\title{
Single-shot picosecond resolution Fourier transform holographic microscopy with large field of view using a compact soft $\mathrm{x}$-ray laser
}

Shoujun Wang, Alex Rockwood, Yong Wang, Wei-Lun Chao, Patrick Naulleau, et al.

Shoujun Wang, Alex Rockwood, Yong Wang, Wei-Lun Chao, Patrick Naulleau, Huanyu Song, Carmen S. Menoni, Mario Marconi, Jorge J. Rocca, "Single-shot picosecond resolution Fourier transform holographic microscopy with large field of view using a compact soft x-ray laser," Proc. SPIE 11886, International Conference on X-Ray Lasers 2020, 1188600 (8 July 2021); doi: $10.1117 / 12.2594832$

SPIE. Event: XVII International Conference on X-Ray Lasers, 2020, Online Only 


\title{
Single-shot picosecond resolution Fourier transform holographic microscopy with large field of view using a compact soft $X$-ray laser
}

\author{
Shoujun Wang*a ${ }^{* a}$ Alex Rockwood ${ }^{\mathrm{b}}$, Yong Wang ${ }^{\mathrm{a}}$, Wei-Lun Chao ${ }^{\mathrm{c}}$, Patrick Naulleauc ${ }^{\mathrm{c}}$, Huanyu \\ Song ${ }^{\mathrm{a}}$, Carmen S. Menoni ${ }^{\mathrm{a}}$, Mario Marconi ${ }^{\mathrm{a}}$, and Jorge J. Rocca ${ }^{\mathrm{a}, \mathrm{b}}$ \\ ${ }^{a}$ Department of Electrical and Computer Engineering, Colorado State University, Fort Collins, \\ Colorado 80523, USA; ${ }^{b}$ Department of Physics, Colorado State University, Fort Collins, Colorado \\ 80523, USA; ' Center for X-Ray Optics, Lawrence Berkeley Laboratory, Berkeley, California 94720, \\ USA
}

\begin{abstract}
We demonstrate single-shot Fourier transform holography with a $7 \mu \mathrm{m}$ diameter field of view and picosecond time resolution using a highly coherent $\sim 5$ ps pulse duration tabletop Ni-like molybdenum soft X-ray laser at $18.9 \mathrm{~nm}$ wavelength. The essentially full spatial coherence soft X-ray source with close to diffraction-limited divergence was implemented utilizing a dual-plasma amplifier scheme. The high flux illumination of $\sim 2 \times 10^{11}$ photons per pulse was split using a Fresnel zone plate to generate the object and reference beams creating high contrast Fourier transform holograms. The final image was numerically reconstructed by 2D Fourier transform. A minimum half-pitch spatial resolution of 62 nm was obtained.
\end{abstract}

Keywords: Holography, X-ray lasers, X-ray imaging, nanotechnology

\section{INTRODUCTION}

Disciplines with high societal impact like material science, biology, medicine etc. relies on sophisticated imaging techniques for regular diagnostics and research. In fact, the significant advances in high resolution imaging produced in the last decades had potentiated several aspects of these disciplines and opened new fields like for example those associated with nanotechnology. To reach nanoscale spatial resolutions it is customary to utilize charged particles beams in electron and ion microscopes that are capable to produce astonishing high-resolution images reaching even the molecular scale. However, the utilization of charged particles sometimes makes this method unsuitable for some applications due to the interaction of the charged particles with the sample.

Utilization of photons can overcome this limitation, offering also the possibility of ultrafast time resolution. Optical microscopy is directly associated with the advancement of science in the last centuries, and nowadays it is customary to use microscopes in almost all scientific disciplines. In the optical realm, the achievable spatial resolution is directly proportional to the wavelength of the illumination. Thus, one strategy to improve the resolution is to reduce the wavelength of the illumination and improve the resolution proportionally. A particular spectral range well suited to image nanostructures is the extreme ultraviolet (EUV) and soft x-ray (SXR) region. In this spectral range, there are available several accelerator-based national facilities ${ }^{1-4}$, as well as by more compact sources ${ }^{5-10}$ capable to deliver enough photon flux to attempt high resolution images on nanoscale specimens efficiently. In particular, as discussed below, soft x-ray lasers produce a large number of photons in a single pulse of picosecond duration allowing for ultrafast single-shot imaging of dynamic phenomena in the nanoscale.

The availability of these short wavelength sources motivated extensive work developing full field microscopes using zone plate (ZP) lenses. Of particular impact are the microscopes implemented with compact plasmas-based discharge pumped $^{11,12}$ and laser-pumped ${ }^{13-15}$ EUV/SXR lasers, that achieved resolutions down to $38 \mathrm{~nm}$ resolution in transmission mode ${ }^{15}$ and $55 \mathrm{~nm}$ in reflection mode ${ }^{14}$. Also, stroboscopic imaging of oscillating magnetic probes in the EUV using ZPs was demonstrated with $\sim 1 \mathrm{~ns}$ temporal resolution and $54 \mathrm{~nm}$ spatial resolution using a $46.9 \mathrm{~nm}$ capillary discharge laser ${ }^{16}$. Instead of using ZP optics, an alternative approach very well adapter to the EUV is to eliminate optical components all together and measure the far field diffraction pattern of the object. This is the approach of Fourier transform holography $(\mathrm{FTH})^{17}$ and coherent diffractive imaging $(\mathrm{CDI})^{18}$. However, the absence of optics that gather the light into the image plane 
imposes for these approaches the challenge to obtain enough flux to be able to record single-shot images with high spatial and temporal resolution ${ }^{12,16,19-24}$.

The FTH hologram is recorded as the interference between the diffracted wave by the object and a point source reference wave. The image is then numerically reconstructed simply via a 2D Fast Fourier Transformation (FFT) of the hologram, making FTH an ideal method for high-resolution real-time imaging. In FTH there are several factors that determine the spatial resolution and the field of view (FOV). In the reconstruction process, the image is obtained as the convolution of the object and the point source reference beams. As such, the resolution in the reconstructed image depends on the size of the reference beam, this is how close the reference source is to a delta function-like reference.

Requiring small size refence beam, imposes the immediate challenge in the fabrication technique to create a small pinhole aperture. In turn, a small pinhole restricts dramatically the intensity of the reference beam, producing interference fringes with very poor visibility if the object and reference beams have very dissimilar intensities. As a consequence, the low flux requires a long exposure time to increase the hologram signal-to-noise ratio (SNR). Anyway, there are some clever means to partially overcome this inconvenience. An alternative setup to improve the SNR is utilizing multiple reference sources, to extend the detection limit ${ }^{25}$. Replacing the reference pinhole with a uniformly redundant array also showed improvement in the resolutions to $44 \mathrm{~nm}^{26}$. On the other hand, to image a large object it is necessary to have a large FOV, which generates high spatial frequency fringes. Consequently, the numerical aperture and the detector's pixel size limit the maximum spatial frequency that can be recorded and therefore sets a limit on the spatial resolution. Extended FOV FTH spanning $180 \mu \mathrm{m}$ has been demonstrated by applying spatial multiplexing using a filtered soft X-ray beam originating from an undulator source ${ }^{27}$. Such full-field of view imaging needs long exposure time and/or a large photon flux that is only achievable at synchrotrons and Free-Electron Lasers (FELs) ${ }^{28}$.

Table-top EUV coherent sources have the unique advantage to enable a more readily access to experiments, as compared with large EUV time-shared facilities. The successful demonstration of an X-ray nanoscale imaging with high spatial and temporal resolution in a large FOV at table-top scale undoubtedly will provide an attractive tool that can have a significant impact. Such compact table-top system could use sources that include high harmonic generation (HHG) and plasma-based SXR lasers.

With HHG CDI has been successfully demonstrated at table-top scale ${ }^{29,30}$ with a best resolution of $22 \mathrm{~nm}^{31}$, but in a single shot a resolution of $119 \mathrm{~nm}$ was reported [20]. FTH using a HHG source also been demonstrated with a resolution of 53 $\mathrm{nm}^{32}$. However, the limited photon flux per-pulse of HHG sources requires long exposure times. For the same reason, the imaged area was limited to less than 9 square micrometers. Recently by increasing HHG repetition rate to $100 \mathrm{kHz}$, a halfpitch resolution of $34 \mathrm{~nm}$ was obtained with a reduced exposure time of tens of seconds. In this case, the imaged object area was only $0.075 \mu^{2}{ }^{33}$

Plasma-based SXR lasers provide a much larger number, $\sim 10^{11}$, photons per-pulse, which in principle is a clear advantage over HHG sources for high temporal resolution (single-shot) FTH recording. SXR and EUV holograms have a very prolific history. The first hologram obtained with a plasma based SXR laser was a Gabor hologram ${ }^{34}$ more than 30 years ago. More recently a resolution of $87 \mathrm{~nm}$ has been obtained using a FTH setup with a FOV of $9 \mu \mathrm{m}^{2} .{ }^{35}$ Using an EUV capillary discharge laser source operating at $46.9 \mathrm{~nm}$ wavelength a spatial resolution of $169 \mathrm{~nm}$ was obtained in a single laser pulse with temporal resolution of $\approx 1 \mathrm{~ns}{ }^{21,36}$. Single-shot CDI with $180 \mathrm{~nm}$ resolution using an $18.9 \mathrm{~nm}$ Ni-like Mo SXR laser was also reported ${ }^{37}$.

In this work, we implemented a single-shot picosecond resolution FTH using a setup that a custom-made ZP with a central aperture that plays the role of a beam splitter, similar to the experimental setup used with a synchrotron source ${ }^{17}$. The ZP generates both the point source for the reference source (third order focus) and the illuminating (central opening) beams. In contrast to FTH in which a pinhole produces a reference beam, this geometry increases by orders of magnitude the flux and therefore the area of the object that can be recorded by equalizing the intensities of the object and reference beams. Moreover, by employing a dual-plasma amplifier laser scheme the spatial coherence and beam divergence of the SXR lasers source are dramatically improved. A large number of coherent photons were concentrated on the ZP area generating high contrast holograms which is essential for single-shot large FOV FTH. A half-pitch resolution of up to $62 \mathrm{~nm}$ was achieved with single-shot SXR laser illumination, yielding a temporal resolution of 5 ps. The area imaged was $38.5 \mu m^{2}$ i.e. near an order of magnitude larger than formerly reported experiments with SXR lasers ${ }^{35}$ and more than 500 times the area in experiments using HHG sources ${ }^{33}$. 


\section{EXPERIMENTAL SETUP}

The experiment was conducted at Colorado State University's ALEPH laser facility. A high quality SXR coherent beam is essential to demonstrate the ideal of large FOV FTH in a single shot. To improve the spatial coherent of the SXR laser source the self-seeded dual-plasma configuration ${ }^{38}$ was employed. Particularly two spatially separated plasma columns were pumped by two sets of beams coming from same laser with adequate delay intervals. The small cross-section area of the second plasma gain region acts as a spatial filter that filtering only small fraction of the amplified spontaneous emission from the first plasma amplifier. This filtered fraction, about $20 \mu \mathrm{m}$ in diameter, has good wavefront and spatial coherent is going to be further amplified. We chose Ni-like Mo SXR laser as the source. The generated dual-plasma self-seeded SXR laser beam has a near-diffraction limited beam divergence of $0.66 \mathrm{mrad}$ and nearly full transverse coherent with up to $\sim 2 \times 10^{11}$ photons per pulse at $\lambda=18.9 \mathrm{~nm}^{39}$. The pulse duration of this type of Ni-like collisionally excited grazing incidence plasma-based SXR lasers was measured to be $\sim 5 \mathrm{ps}$ using a streak camera ${ }^{40}$. This highly coherent bright SXR beam was directed by a flat gold coated mirror at grazing incidence to the FTH experiment.

Figure 1 shows the Fourier holography experiment setup. Ni-like Mo SXR laser illuminates a custom-made condenser ZP with a central aperture that plays the role of a beam splitter generating both the point reference source (third order focus) and the illuminating (central opening) beams. The SXR laser beam passes through the central aperture of the ZP and illuminates the object located in a downstream plane. The ZP focuses the remaining part of the beam into a small pinhole located in the same plane of the object creating the FTH reference beam. Here the pinhole is designed to block other diffraction orders of the ZP, while the spatial resolution defined by the ZP focal spot size. The scattered beam from the object and the reference beam interfere in a CCD camera that records the hologram. The size of the object hole and its distance from the reference hole was selected to assure the interference fringes are resolved at the CCD and there is no superposition between autocorrelation and cross correlation in the FFT reconstruction.

The use of a ZP can gather a larger number of photos of the illuminating beam and better equalize the intensity between the object and the reference beams, which in turn overcomes the object size limitations in the mask-based FTH. Another advantage is the ZP design can be optimized to generate a tens of nanometer diameter focal spot. In contrast to FTH in which a pinhole produces a reference beam, this geometry increases by orders of magnitude the flux and therefore the area of the object that can be recorded. The ZP used in this experiment is an available ZP designed for $46.9 \mathrm{~nm}$ wavelength with self-standing structure that allows for maximum transparency of the SXR laser beam. It contains 623 self-standing zones with an outer diameter of $500 \mu \mathrm{m}$, an outer zone width of $200 \mathrm{~nm}$ and a center aperture of $30 \mu \mathrm{m}$. In this work we use the 3rd order of the ZP which gives a calculated spot size of $81 \mathrm{~nm}$, a NA of 0.14 and a focal length of $1.78 \mathrm{~mm}$ for $\lambda=18.9 \mathrm{~nm}$. By using a higher diffraction order, it is possible to achieve a smaller focal spot and larger NA, increasing the FTH resolution.

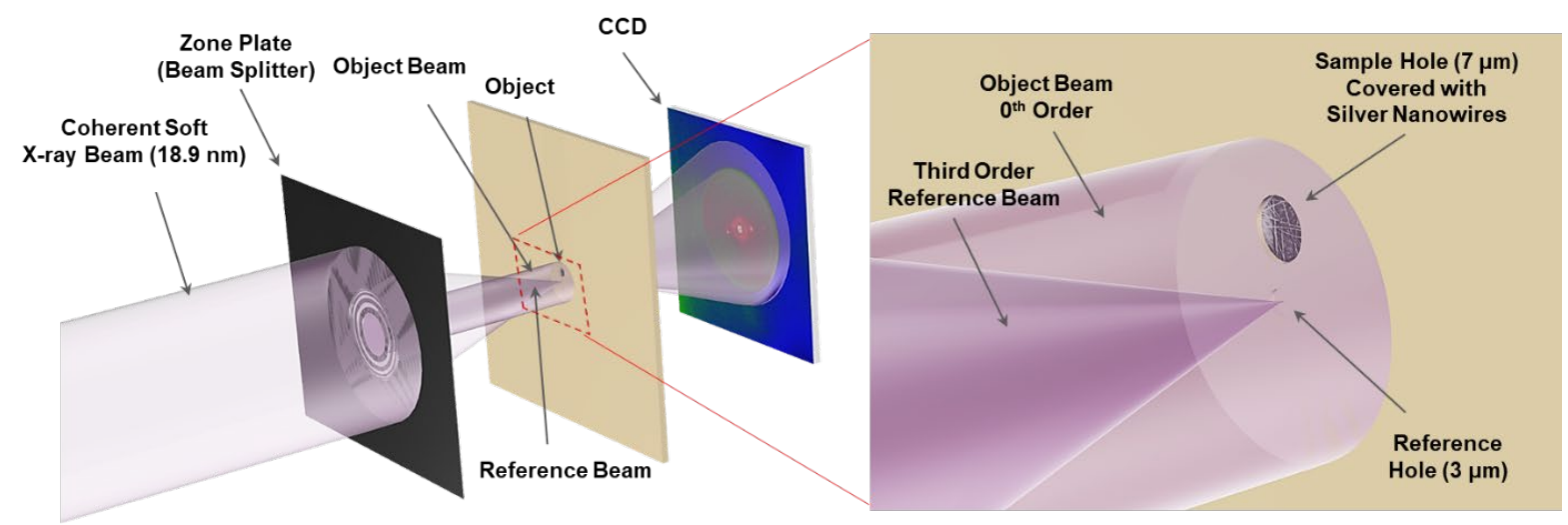

Figure 1. Schematic setup of Fourier holography experiment. A zone plate is used as a beam splitter. The mask contains a hole to define the reference beam and a semitransparent area to support the sample. The hologram is recorded by a CCD camera. A zoomed-in inset shows the beam that illuminates the sample and the refence beam. 
The object was placed at the focal distance of $1.78 \mathrm{~mm}$ away from the ZP. The sample holder was implemented with a 50 $\mathrm{nm}$ thick $\mathrm{Si}_{2} \mathrm{~N}_{3}$ membrane with coatings to enhance the absorption. A $7 \mu$ m diameter object region was left without coating to preserve a region with higher transmission for the beam. The Ag nanowire samples, diameter varied from $180 \mathrm{~nm}$ to $120 \mathrm{~nm}$, are randomly spin coated over the region. Additionally, a $3 \mu \mathrm{m}$ hole was defined in the membrane to allow for the passage of the reference beam produced by the focus of the ZP. The pinhole for the reference beam was located at 8 $\mu \mathrm{m}$ from the center of the sample region. Figure 2 shows two samples images from Scanning electron microscope (SEM) that prepared for the experiment. The CCD camera with the pixel size of $13.5 \mu \mathrm{m}$ and $2048 \times 2048$ pixels placed $50 \mathrm{~mm}$ away from the sample to capture the diffracted light.
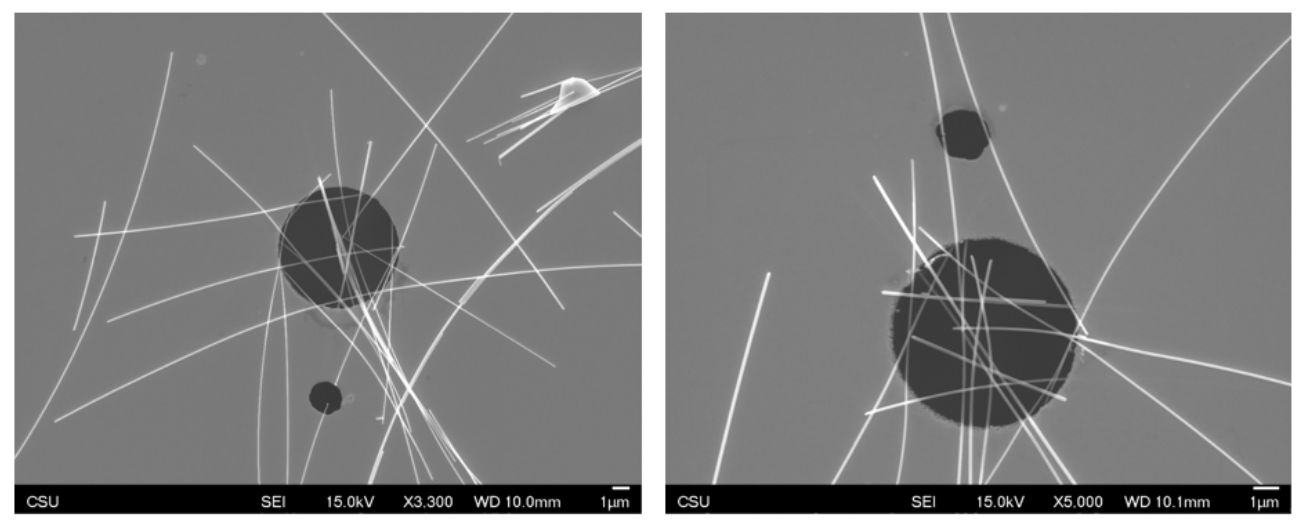

Figure. 2. SEM images of the two samples used in the experiment. Each sample consists of an opaque membrane with a $7 \mu \mathrm{m}$ hole where silver nanowires were placed. The membrane contains a $3 \mu \mathrm{m}$ diameter hole for the reference beam separated by a center-to-center distance of $\sim 8 \mu \mathrm{m}$.

\section{RESULTS}

A typical single-shot hologram and its FFT reconstruction are shown in Figure 3. The raw hologram data was further processed to reduce the influence of the noise. An FFT of the data finally resulted in an image in the object at the plane of the ZP focus. By zooming in on the area of interest the nanowires are clearly visible. Figure 4 compares picosecond resolution single-shot images of two nanowire samples to those obtained from the same samples using a scanning electron microscope.

(a)

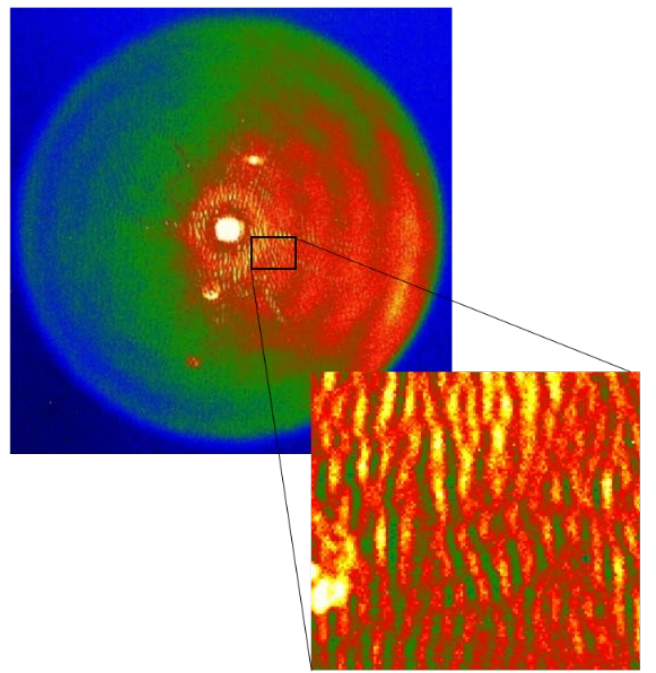

(b)

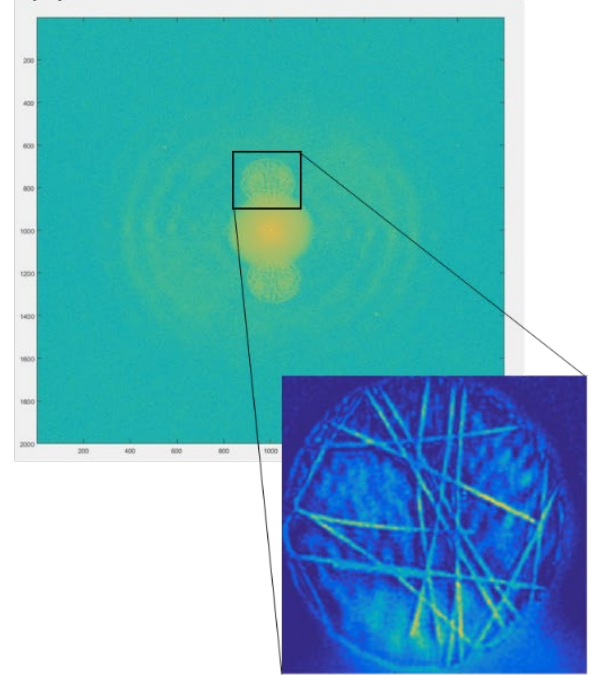

Figure 3. (a) Typical single shot SXR laser Fourier holography image with a zoom of the fringe pattern. (b) The Fourier transform of the hologram with zoomed-in inset showing Ag nanowire object. 

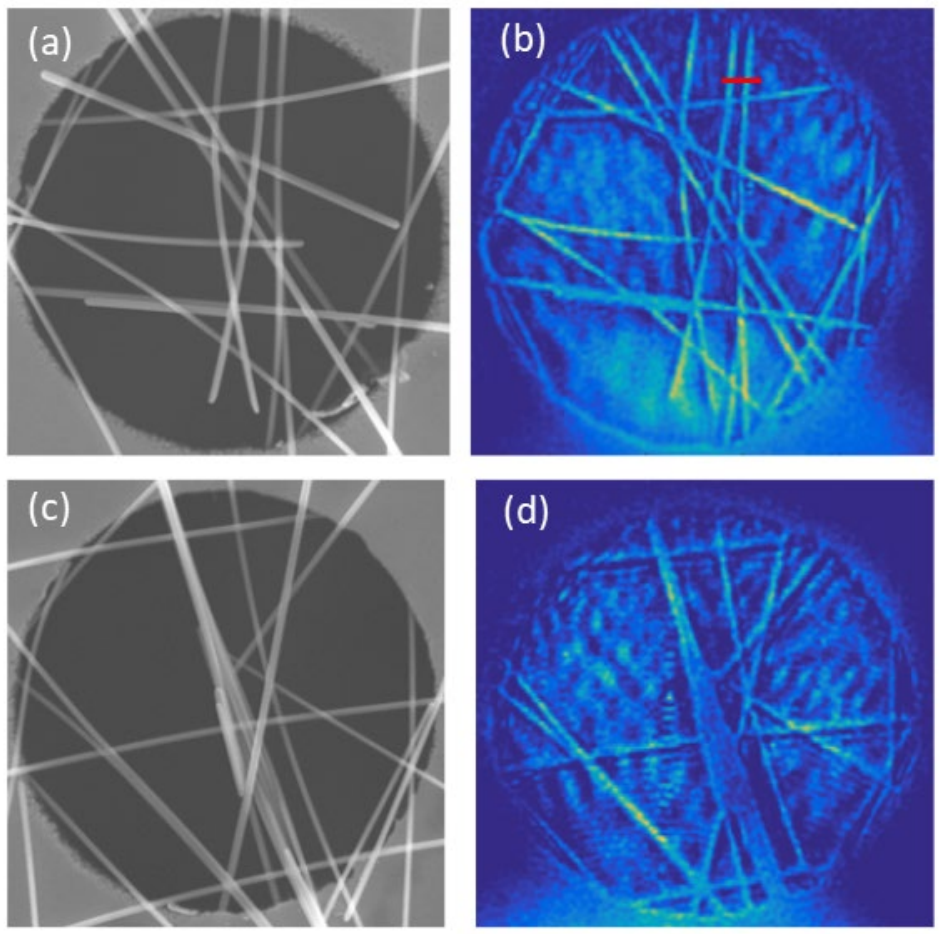

Figure 4. Electron microscope image (a), (c) and corresponding single shot holographic images (b), (d) of the silver nanowires over a $7 \mu \mathrm{m}$ diameter hole.

The $10 \%$ to $90 \%$ knife edge criterium was used to assess the spatial resolution. The reconstruction of the image is the results of the convolution of the object with a point source. Here we assume the source has a Gaussian with FWHM of 81 $\mathrm{nm}$. Convolving a Gaussian function with a step function, produces also a Gaussian profile. This Gaussian profile is the image that we can expect to obtain after the reconstruction from a hard-edge object. The spatial spread for an $81 \mathrm{~nm}$ FWHM Gaussian to rise from $10 \%$ to $90 \%$ of its peak value is $\Delta x=58 \mathrm{~nm}$. It is consistent with the experimental results we obtained of $62 \mathrm{~nm}$ which shows in Figure 5 as a typical cross section image, corresponding to the small red segment of $\mathrm{Ag}$ nanowires indicated in Figure 4(b). 


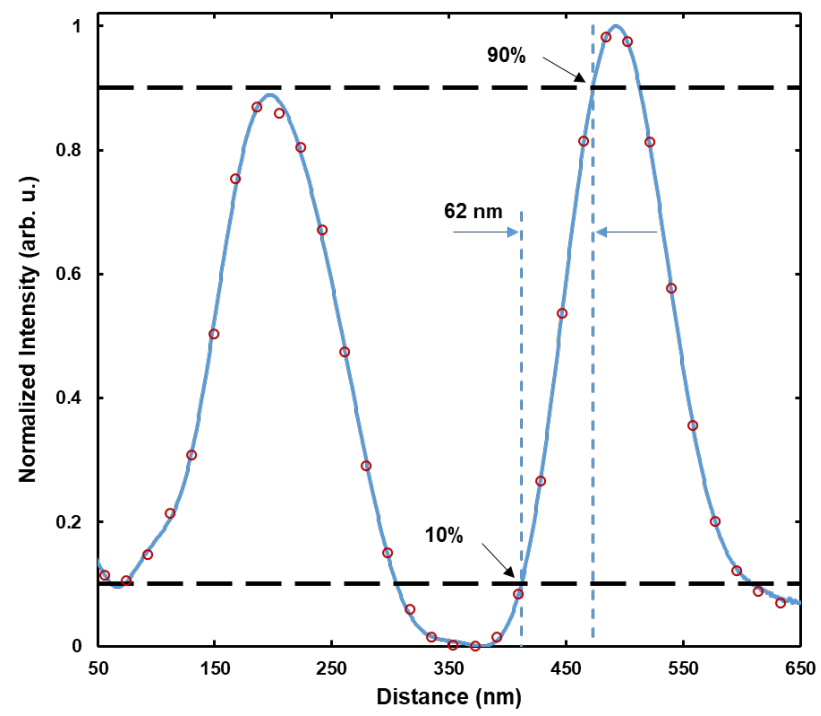

Figure 5. Characteristic $10 \%$ to $90 \%$ knife edge cut of the image showing a spatial resolution of $62 \mathrm{~nm}$.

The utilization of the ZP to implement the point reference source allowed for an extended field of view of $38.5 \mu \mathrm{m}^{2}$. The imaged area could be further increased without sacrificing resolution by enlarging the high transmission area where the sample is located while maintaining its distance to the reference pinhole. This could be achieved using to a half doughnut shape instead of the circle. The area could also be enlarged by increasing the size of the central opening on the ZP, to create a larger illuminated area. Finally, since the reconstruction of the hologram produces an image of the object at the plane where the reference source is located, it would be possible to inspect the object at different planes along the optical axis by displacing the $\mathrm{ZP}$, which will enable one to compose a tomography of the object. With the recent demonstration of sub $8 \mathrm{~nm}$ wavelength gain-saturated plasma-based table-top SXR laser ${ }^{10}$ it will be possible to further improve the resolutions both in time and space using compact table-top systems.

\section{Acknowledgment}

The work was supported by the AMOS program of the Office of Basic Energy Sciences, US Department of Energy grant number (DE-FG02-04ER15592). Previous support from DARPA grant D16PC00087 is acknowledged. The experiments were conducted at CSU's ALEPH laser facility supported by LaserNet US (DE-SC0019076).

\section{REFERENCES}

[1] Mcneil, B. W. J. and Thompson, N. R., "X-ray free-electron lasers," Nat. Photonics 4(12), 814-821 (2010).

[2] Faatz, B., Baboi, N., Ayvazyan, V., Balandin, V., Decking, W., Duesterer, S., Eckoldt, H. J., Feldhaus, J., Golubeva, N., Koerfer, M., Laarmann, T., Leuschner, A., Lilje, L., Limberg, T., Noelle, D., Obier, F., Petrov, A., Ploenjes, E., Rehlich, K., et al., "FLASH II: A seeded future at FLASH,” IPAC 2010 - 1st Int. Part. Accel. Conf., 2152-2154 (2010).

[3] Patterson, B. D., Abela, R., Braun, H.-H., Flechsig, U., Ganter, R., Kim, Y., Kirk, E., Oppelt, A., Pedrozzi, M., Reiche, S., Rivkin, L., Schmidt, T., Schmitt, B., Strocov, V. N., Tsujino, S. and Wrulich, A. F., "Coherent science at the SwissFEL x-ray laser," New J. Phys. 12(3), 35012 (2010).

[4] Allaria, E., Badano, L., Bassanese, S., Capotondi, F., Castronovo, D., Cinquegrana, P., Danailov, M. B., D’Auria, G., Demidovich, A., De Monte, R., De Ninno, G., Di Mitri, S., Diviacco, B., Fawley, W. M., Ferianis, M., Ferrari, E., Gaio, G., Gauthier, D., Giannessi, L., et al., "The FERMI free-electron lasers," J. Synchrotron Radiat. 22(3), 485-491 (2015).

[5] Chatziathanasiou, S., Kahaly, S., Skantzakis, E., Sansone, G., Lopez-Martens, R., Haessler, S., Varju, K., 
Tsakiris, G. D., Charalambidis, D. and Tzallas, P., "Generation of attosecond light pulses from gas and solid state media," Photonics 4(2) (2017).

[6] Rocca, J. J., “Table-top soft x-ray lasers,” Rev. Sci. Instrum. 70(10), 3799-3827 (1999).

[7] Wang, Y., Granados, E., Pedaci, F., Alessi, D., Luther, B., Berrill, M. and Rocca, J. J., "Phase-coherent, injection-seeded, table-top soft-X-ray lasers at $18.9 \mathrm{~nm}$ and $13.9 \mathrm{~nm}$," Nat. Photonics 2(2), 94-98 (2008).

[8] Alessi, D., Wang, Y., Luther, B. M., Yin, L., Martz, D. H., Woolston, M. R., Liu, Y., Berrill, M. and Rocca, J. J., "Efficient Excitation of Gain-Saturated Sub-9-nm-Wavelength Tabletop Soft-X-Ray Lasers and Lasing Down to 7.36 nm," Phys. Rev. X 1(2) (2011).

[9] Depresseux, A., Oliva, E., Gautier, J., Tissandier, F., Nejdl, J., Kozlova, M., Maynard, G., Goddet, J. P., Tafzi, A., Lifschitz, A., Kim, H. T., Jacquemot, S., Malka, V., Ta Phuoc, K., Thaury, C., Rousseau, P., Iaquaniello, G., Lefrou, T., Flacco, A., et al., "Table-top femtosecond soft X-ray laser by collisional ionization gating," Nat. Photonics 9(12) (2015).

[10] Rockwood, A., Wang, Y., Wang, S., Berrill, M., Shlyaptsev, V. N. and Rocca, J. J., "Compact gain-saturated xray lasers down to $6.85 \mathrm{~nm}$ and amplification down to $5.85 \mathrm{~nm}$," Optica 5(3), 257-262 (2018).

[11] Vaschenko, G., Brizuela, F., Brewer, C., Grisham, M., Mancini, H., Menoni, C. S., Marconi, M. C., Rocca, J. J., Chao, W., Liddle, J. A., Anderson, E. H., Attwood, D. T., Vinogradov, A. V., Artioukov, I. A., Pershyn, Y. P. and Kondratenko, V. V., "Nanoimaging with a compact extreme-ultraviolet laser," Opt. Lett. 30(16) (2005).

[12] Brewer, C. A., Brizuela, F., Wachulak, P., Martz, D. H., Chao, W., Anderson, E. H., Attwood, D. T., Vinogradov, A. V., Artyukov, I. A., Ponomareko, A. G., Kondratenko, V. V., Marconi, M. C., Rocca, J. J. and Menoni, C. S., "Single-shot extreme ultraviolet laser imaging of nanostructures with wavelength resolution," Opt. Lett. 33(5) (2008).

[13] Brizuela, F., Wang, Y., Brewer, C. A., Pedaci, F., Chao, W., Anderson, E. H., Liu, Y., Goldberg, K. A., Naulleau, P., Wachulak, P., Marconi, M. C., Attwood, D. T., Rocca, J. J. and Menoni, C. S., "Microscopy of extreme ultraviolet lithography masks with $13.2 \mathrm{~nm}$ tabletop laser illumination," Opt. Lett. 34(3) (2009).

[14] Brizuela, F., Carbajo, S., Sakdinawat, A., Alessi, D., Martz, D. H., Wang, Y., Luther, B., Goldberg, K. A., Mochi, I., Attwood, D. T., La Fontaine, B., Rocca, J. J. and Menoni, C. S., "Extreme ultraviolet laser-based table-top aerial image metrology of lithographic masks," Opt. Express 18(14), 14467-14473 (2010).

[15] Vaschenko, G., Brewer, C., Brizuela, F., Wang, Y., Larotonda, M. A., Luther, B. M., Marconi, M. C., Rocca, J. J., Menoni, C. S., Anderson, E. H., Chao, W., Harteneck, B. D., Liddle, J. A., Liu, Y. and Attwood, D. T., "Sub$38 \mathrm{~nm}$ resolution tabletop microscopy with $13 \mathrm{~nm}$ wavelength laser light," Opt. Lett. 31(9), 1214-1216 (2006).

[16] Carbajo, S., Howlett, I. D., Brizuela, F., Buchanan, K. S., Marconi, M. C., Chao, W., Anderson, E. H., Artioukov, I., Vinogradov, A., Rocca, J. J. and Menoni, C. S., "Sequential single-shot imaging of nanoscale dynamic interactions with a table-top soft x-ray laser," Opt. Lett. 37(14), 2994-2996 (2012).

[17] McNulty, I., Kirz, J., Jacobsen, C., Anderson, E. H., Howells, M. R. and Kern, D. P., "High-resolution imaging by fourier transform X-ray holography," Science (80-. ). (1992).

[18] Miao, J., Charalambous, P., Kirz, J. and Sayre, D., "Extending the methodology of X-ray crystallography to allow imaging of micrometre-sized non-crystalline specimens," Nature (1999).

[19] Barty, A., Boutet, S., Bogan, M. J., Hau-Riege, S., Marchesini, S., Sokolowski-Tinten, K., Stojanovic, N., Tobey, R., Ehrke, H., Cavalleri, A., Düsterer, S., Frank, M., Bajt, S., Woods, B. W., Seibert, M. M., Hajdu, J., Treusch, R. and Chapman, H. N., "Ultrafast single-shot diffraction imaging of nanoscale dynamics," Nat. Photonics 2(7) (2008).

[20] Ravasio, A., Gauthier, D., Maia, F. R. N. C., Billon, M., Caumes, J. P., Garzella, D., Géléoc, M., Gobert, O., Hergott, J. F., Pena, A. M., Perez, H., Carré, B., Bourhis, E., Gierak, J., Madouri, A., Mailly, D., Schiedt, B., Fajardo, M., Gautier, J., et al., "Single-shot diffractive imaging with a table-top femtosecond soft X-ray laserharmonics source," Phys. Rev. Lett. 103(2) (2009).

[21] Monserud, N. C., Malm, E. B., Wachulak, P. W., Putkaradze, V., Balakrishnan, G., Chao, W., Anderson, E., Carlton, D. and Marconi, M. C., "Recording oscillations of sub-micron size cantilevers by extreme ultraviolet Fourier transform holography," Opt. Express (2014).

[22] Gorkhover, T., Ulmer, A., Ferguson, K., Bucher, M., Maia, F. R. N. C., Bielecki, J., Ekeberg, T., Hantke, M. F., Daurer, B. J., Nettelblad, C., Andreasson, J., Barty, A., Bruza, P., Carron, S., Hasse, D., Krzywinski, J., Larsson, D. S. D., Morgan, A., Mühlig, K., et al., "Femtosecond X-ray Fourier holography imaging of free-flying nanoparticles," Nat. Photonics 12(3), 150-153 (2018).

[23] Tenboer, J., Basu, S., Zatsepin, N., Pande, K., Milathianaki, D., Frank, M., Hunter, M., Boutet, S., Williams, G. J., Koglin, J. E., Oberthuer, D., Heymann, M., Kupitz, C., Conrad, C., Coe, J., Roy-Chowdhury, S., Weierstall, 
U., James, D., Wang, D., et al., "Time-resolved serial crystallography captures high-resolution intermediates of photoactive yellow protein," Science (80-. ). 346(6214) (2014).

[24] Lai, Y., Xue, Y., Côté, C. Y., Liu, X., Laramée, A., Jaouen, N., Légaré, F., Tian, L. and Liang, J., "Single-Shot Ultraviolet Compressed Ultrafast Photography," Laser Photonics Rev. 14(10) (2020).

[25] Schlotter, W. F., Rick, R., Chen, K., Scherz, A., Stöhr, J., Lüning, J., Eisebitt, S., Günther, C., Eberhardt, W., Hellwig, O. and McNulty, I., "Multiple reference Fourier transform holography with soft x rays," Appl. Phys. Lett. (2006).

[26] Marchesini, S., Boutet, S., Sakdinawat, A. E., Bogan, M. J., Bajt, S., Barty, A., Chapman, H. N., Frank, M., HauRiege, S. P., Szöke, A., Cui, C., Shapiro, D. A., Howells, M. R., Spence, J. C. H., Shaevitz, J. W., Lee, J. Y., Hajdu, J. and Seibert, M. M., "Massively parallel X-ray holography," Nat. Photonics (2008).

[27] Schlotter, W. F., Lüning, J., Rick, R., Chen, K., Scherz, A., Eisebitt, S., Günther, C. M., Eberhardt, W., Hellwig, O. and Stöhr, J., "Extended field of view soft x-ray Fourier transform holography: toward imaging ultrafast evolution in a single shot," Opt. Lett. 32(21) (2007).

[28] Pfau, B., Günther, C. M., Schaffert, S., Mitzner, R., Siemer, B., Roling, S., Zacharias, H., Kutz, O., Rudolph, I., Treusch, R. and Eisebitt, S., "Femtosecond pulse X-ray imaging with a large field of view," New J. Phys. (2010).

[29] Sandberg, R. L., Paul, A., Raymondson, D. A., Hädrich, S., Gaudiosi, D. M., Holtsnider, J., Tobey, R. I., Cohen, O., Murnane, M. M., Kapteyn, H. C., Song, C., Miao, J., Liu, Y. and Salmassi, F., "Lensless diffractive imaging using tabletop coherent high-harmonic soft-X-ray beams," Phys. Rev. Lett. 99(9) (2007).

[30] Sandberg, R. L., Song, C., Wachulak, P. W., Raymondson, D. A., Paul, A., Amirbekian, B., Lee, E., Sakdinawat, A. E., La-O-Vorakiat, C., Marconi, M. C., Menoni, C. S., Murnane, M. M., Rocca, J. J., Kapteyn, H. C. and Miao, J., "High numerical aperture tabletop soft x-ray diffraction microscopy with 70-nm resolution," Proc. Natl. Acad. Sci. U. S. A. 105(1) (2008).

[31] Seaberg, M. D., Adams, D. E., Townsend, E. L., Raymondson, D. A., Schlotter, W. F., Liu, Y., Menoni, C. S., Rong, L., Chen, C.-C., Miao, J., Kapteyn, H. C. and Murnane, M. M., "Ultrahigh 22 nm resolution coherent diffractive imaging using a desktop $13 \mathrm{~nm}$ high harmonic source," Opt. Express 19(23) (2011).

[32] Sandberg, R. L., Raymondson, D. a., La-o-vorakiat, C., Paul, A., Raines, K. S., Miao, J., Murnane, M. M., Kapteyn, H. C. and Schlotter, W. F., "Tabletop soft-x-ray Fourier transform holography with $50 \mathrm{~nm}$ resolution," Opt. Lett. (2009).

[33] Tadesse, G. K., Eschen, W., Klas, R., Hilbert, V., Schelle, D., Nathanael, A., Zilk, M., Steinert, M., Schrempel, F., Pertsch, T., Tünnermann, A., Limpert, J. and Rothhardt, J., "High resolution XUV Fourier transform holography on a table top," Sci. Rep. (2018).

[34] TREBES, J. E., BROWN, S. B., CAMPBELL, E. M., MATTHEWS, D. L., NILSON, D. G., STONE, G. F. and WHELAN, D. A., "Demonstration of X-ray Holography with an X-ray Laser," Science (80-. ). 238(4826), 517 LP - 519 (1987).

[35] Kim, H. T., Kim, I. J., Kim, C. M., Jeong, T. M., Yu, T. J., Lee, S. K., Sung, J. H., Yoon, J. W., Yun, H., Jeon, S. C., Choi, I. W. and Lee, J., "Single-shot nanometer-scale holographic imaging with laser-driven X-ray laser," Appl. Phys. Lett. (2011).

[36] Malm, E. B., Monserud, N. C., Brown, C. G., Wachulak, P. W., Xu, H., Balakrishnan, G., Chao, W., Anderson, E. and Marconi, M. C., "Tabletop single-shot extreme ultraviolet Fourier transform holography of an extended object," Opt. Express (2013).

[37] Zürch, M., Jung, R., Späth, C., Tümmler, J., Guggenmos, A., Attwood, D., Kleineberg, U., Stiel, H. and Spielmann, C., "Transverse Coherence Limited Coherent Diffraction Imaging using a Molybdenum Soft X-ray Laser Pumped at Moderate Pump Energies," Sci. Rep. (2017).

[38] Nishikino, M., Tanaka, M., Nagashima, K., Kishimoto, M., Kado, M., Kawachi, T., Sukegawa, K., Ochi, Y., Hasegawa, N. and Kato, Y., "Demonstration of a soft-x-ray laser at $13.9 \mathrm{~nm}$ with full spatial coherence," Phys. Rev. A - At. Mol. Opt. Phys. 68(6), 4 (2003).

[39] Wang, S., Rockwood, A., Wang, Y., Chao, W.-L., Naulleau, P., Song, H., Menoni, C. S., Marconi, M. and Rocca, J. J., "Single-shot large field of view Fourier transform holography with a picosecond plasma-based soft X-ray laser," Opt. Express 28(24) (2020).

[40] Larotonda, M. A., Wang, Y., Berrill, M., Luther, B. M., Rocca, J. J., Shakya, M. M., Gilbertson, S. and Chang, Z., "Pulse duration measurements of grazing-incidence-pumped high repetition rate Ni-like $\mathrm{Ag}$ and $\mathrm{Cd}$ transient soft x-ray lasers," Opt. Lett. (2006). 